\title{
Microanalysis Characterization of Bioactive Protein- Bound Polysaccharides Produced by Amanita Ponderosa Cultures
}

\author{
Cátia Salvador, ${ }^{1,2,4}$ M. Rosário Martins, ${ }^{1,3,4}$ and A. Teresa Caldeira ${ }^{1,2,4, *}$ \\ ${ }^{1}$ Chemistry Department, University of Évora, 7000-671 Évora, Portugal \\ ${ }^{2}$ Chemistry Centre of Évora, University of Évora, 7000-671 Évora, Portugal \\ ${ }^{3}$ Institute of Mediterranean Agricultural and Environmental Sciences, University of Évora, 7002-554 Évora, Portugal \\ ${ }^{4}$ Hercules Laboratory, University of Évora, 7000-809 Évora, Portugal
}

\begin{abstract}
Different compounds of edible mushrooms are responsible for their bioactivity. The ability to synthesize polysaccharides, namely protein-polysaccharide (PPS) complexes, is related to the antioxidant capacity of these compounds and present great interest in preventing a number of diseases, including cancer, cardiovascular and auto-immune diseases, and accelerated aging. Amanita ponderosa are wild edible mushrooms that grow in Mediterranean "montado" areas [Portuguese name given to cork oak (Quercus suber) and holm oak (Quercus ilex) forests]. The aim of this study was to evaluate the production of PPS complexes obtained from A. ponderosa cultures using a new microanalytical approach to quickly and easily monitor the production process. Microanalysis using Fourier-transform infrared using attenuated total reflection and Raman spectroscopy of PPS samples showed spectra compatible with identification of this type of compound in culture extracts. PPS separated by size-exclusion chromatography showed seven main complexes. Molecular weights of the main PPS complexes isolated from cultures ranged between 1.5 and $20 \mathrm{kDa}$ and did not present toxicity against Artemia salina, demonstrating the potential of $A$. ponderosa as a source of biologically active compounds with nutraceutical value. Application of this microanalytical approach to monitoring the production of PPS compounds can be successfully applied in biotechnological processes.
\end{abstract}

Key words: Amanita ponderosa, edible mushrooms, protein-bound polysaccharides, FTIR-ATR, Raman size-exclusion chromatography

\section{INTRODUCTION}

Polysaccharides widely exist in plants, microorganisms (fungi and bacteria), algae, and animals. Together with proteins and polynucleotides, they are essential biomacromolecules in life activities and play important roles in cell-cell communication, cell adhesion, and molecular recognition in the immune system. In recent years, some bioactive polysaccharides isolated from natural sources have attracted much attention in the field of biochemistry and pharmacology (Yang \& Zhang, 2009).

Fungi from the Basidiomycota have received great interest because they contain a large number of biologically active compounds such as polysaccharides, glycoproteins, triterpenes, and antibiotics (Wasser, 2002).

Therefore mushrooms have been used not only as a source of food, but as a medicinal resource as well, and its medicinal properties have been confirmed through intensive research conducted worldwide (Aida et al., 2009; Reis et al., 2012). Currently, mushroom-derived substances with antitumor and immunomodulating properties are used as dietary supplements or functional food for improvement, prevention, and treatment of some diseases (Synytsya et al., 2009; Vaz et al., 2011b).
Different bioactive compounds of edible mushrooms (Barros et al., 2007c, 2008a) are responsible for their antioxidant (Barros et al., 2007a, 2008b, 2008c; Elmastas et al., 2007; Ferreira et al., 2009), antitumoral (Zhang et al., 2007; Cheng et al., 2008; Cheung et al., 2012), antimicrobial (Barros et al., 2007b; Hearst et al., 2009; Rao et al., 2009), antiviral, liver protective (Jayakumar et al., 2006; Lu et al., 2007; Pereira et al., 2012), immunomodulatory (Cui \& Chisti, 2003; Pereira et al., 2012), hypoglycemic, and hypolipidemic properties (Li et al., 2007; Pereira et al., 2012). Antioxidant capacity is clearly associated with the activity of "free radical scavenging enzymes" (superoxide dismutase, catalase, peroxidase) and with the contents of antioxidant substances, mainly phenolic compounds, carotenoids, tocopherol, and ascorbic acid (Elmastas et al., 2007; Ferreira et al., 2007, 2009; Wong \& Chye, 2009).

Different antioxidant compounds such as polysaccharides, ergosterol, nicotinic acid, triterpenes, and phenolic compounds have been found in mushrooms (Cheng et al., 2008; Wong \& Chye, 2009; Vaz et al., 2011a).

In previous studies, polysaccharides have shown important antioxidant properties, presenting antioxidant activity by DPPH radical scavenging in the $\beta$-carotene linoleate model system and by the capacity to mimetize catalase and superoxide dismutase enzymatic activities (Jayakumar et al., 2006; Barros et al., 2008b; Salvador et al., 2012). 